10 Ciccone, E., Pende, D., Vitale, M. et al. (1994) Eur. J. Immuno?. 24, 1003-1009

11 Colonna, M., Spies, T., Strominger, J.L. et al. (1992) Prac. Natl. Acad. Sci. U. S. A. 89, 7983-7985 12 Ciccone, E., Pende, D., Viale, O. et al.
(1992) 1. Exp. Med. 176, 963-971

13 Biassoni, R., Falco, M., Cambiaggi, A. et al. (1995) ]. Exp. Med. 182, 605-609

14 Gumperz, J.E., Litwin, V., Phillips, J.H. et al. (1995) 1. Exp. Med. 181, 1133-1144
15 Döhring, C., Scheidegger, D., Samaridis, J. Cella, M. and Colonna, M. (1996) J. Inmimol. 156, 3098-3103

16 Pende, D., Biassoni, R., Cantoni, C. et al.

(1996) J. Exp. Med. 184, 505-518

\title{
New perspectives on recombinant human antibodies
}

\section{J. de Rruif, A-R. van der Vuurst de Vries, L. Cilenti, E. Boel, W. van Ewijk and T. Logtenberg}

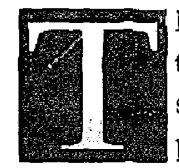

he development of hybridoma technology has generated considerable excitement about the potential use of murine monoclonal antibodies (mAbs) in human immunctherapy. However, clinical testing of these reagents has generally yielded disappointing results: murine mAbs invariably evoke a human anti-mouse immune response and are ineffective as cytotoxic agents and in their ability to recruit effector cells or molecules of the complement system. Unsatisfactory progress in human hybridoma technology has stimulated endeavours aimed at the construction of genetically engineered antibodies with reduced immunogenicity and improved effector functions. To date, such experiments have met with limited success. 'Chimeric' antibodies that combine the variable $(V)$ region of murine antibodies with human immunoglobulin (Ig) constant (C) regions retain their immunogenicity in humans. 'Humanized' antibodies, in which the complementarity-determining regions (CDRs) of murine antibodies are grafted on to a human antibody framework, are tedious to construct and often yield molecules with reduced affinity for antigen. Clearly, these approaches do not provide a robust and reliable scheme for the generation of human antibodies for immunotherapy.

Recent progress in phage-antibodydisplay technology has revolutionized our ability to select human mAbs and construct an array of derivatives with desirable properties for immunotherapy. Large collections ('repertoires') of single chain Fv (scFv) or Fab fragments of human antibodies can be expressed on the surface of filamentous PII: 50167.5699(96)30057.1

\section{The linited porential of murint}

monoclonal antibodie's for hamam

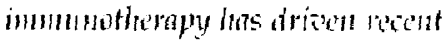

progress in recombinant antibodu tecinology. He're, te Kruit and collengues report on atomes in the deadopment and use of phangeantibody-display libraries.

phage particles, and antibodies with desired specificities can be selected from these phage-display libraries by panning on antigens $^{1,2}$. Surprisingly, this technique has not only proved a powerful means of generating human mAbs against known antigens but, in combination with a variety of subtractive selection methods, also provides a tool to search for novel molecules expressed on the surface of prokaryotic and eukaryotic cells.

\section{Phage-display libraries of human antibody fragments}

A major goal of recombinant-antibody technology is to develop phage-antibody-display libraries of sufficient size and diversity to facilitate isolation of antibodies of every conceivable specificity, including antibodies with high affinity. An important determinant of the composition and diversity of these in vitro immune systems is the source of antibody genes used as building blocks to construct the library.

Libraries can be assembled from the $V$ regions expressed by the $B$ cells of an individual known to have mounted a particular immune response as a result of immurization or exposure to an infectious agent. The B-cell repertoire in the lymphoid organs of these individuals is enriched for antigenspecific $B$ cells and plasma cells that have undergone clonal expansion and antigenaffinity maturation, thus increasing the likelihood of selecting high-affinity antibody fragments from the eventual 'immunized' phage library.

Libraries can also be constructed from the $V$ regions expressed by the $B$ cells of a nonimmunized' individual in an attempt to recruit all the diversity generated by the natural immune system. Importantly, it should be noted that the $B$ cells of naive, immunized or infected individuals have been through positive and negative selection forces in primary and secondary lymphoid organs. The net effect of these processes is a B-cell repertoire that is less diverse than potentially attainable, based on rearrangement of iggene segments, $\mathrm{N}$-region insertions and somatic hypermutation. This in vivo antibodyrepertoire selection is mirrored in the eventual 'naive' phage library.

An alternative approach to create diverse libraries exploits the use of large collections of cloned $\mathrm{V}$ genes to which randomized CDR3 and joining regions $0^{*}$ the heavy chain are fused in vitro by polymerase chain reaction (PCR). The diversity of these 'semi-synthetic' libraries is not constrained by the forces of selection acting in the natural immune system. It may be anticipated that such libraries contain specificities that are not present in the actual antibody repertoires of humans.

Phage-antibody-display libraries of varjous sizes, constructed according to the above mentioned strategies, have been described. As a general rule, it appears that large libraries permit the direct selection of 

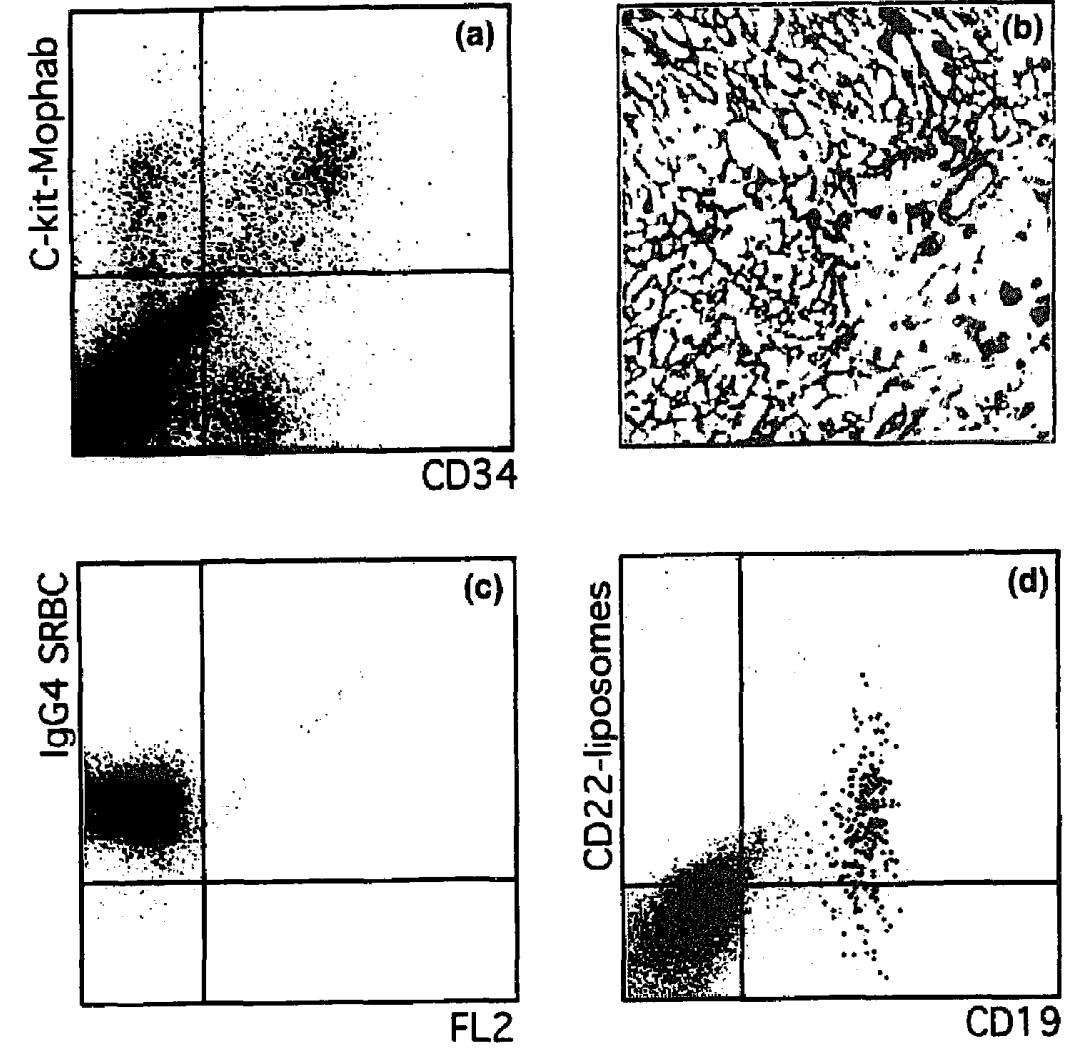

Fig. 1. (a) FACS (fluorescence-actinated cell sorter) analysis of fetal boute marrow cells donblestained with a fhage mondotonal antibody $(\mathrm{mAb})$ specific for $\mathrm{c}$-Kit and a comentional CD34 $\mathrm{mAb}$. (b) Immunuhistochesnical staining of a murine thymus tissue section zuith a monoclonal phage antiboty se'teted for binding to intact fragments of murine thymus. (c) FACS amalysis of sheep red blood cells (SRBCs) stained aith an engineered human IgG4 anfi-SRBC MAb. (d) FACS analysis of blood leukocytes double-staind with n CD19 mAb and CD22-immunoliposomes.

high-affinity antibodies, even from naive or semi-synthetic repertoires ${ }^{1-3}$.

\section{Phage selection strategies}

Phage that express antibody specificities of interest (Phabs) are selected from libraries in a four-step procedure: (1) binding of phages to the target antigen; (2) removal of nonbound phages by washing; (3) elution of bound phages; and (4) infection and propagation of eluted phages in Escherichia coli. Phage selections have been extensively performed on purified antigens coated to plastic surfaces. Although Phabs obtained throngh this procedure perform well in ELISA, their effectiveness in other assays with antigens in their native conformation is usually limited. Presumably the coating procedure leads to the partial destruction of the conformational integrity of $t \mathrm{j}$. atigen and the subsequent selection of Phabs against neo-epitopes. This problem can be circumvented by capturing the target antigen to a solid phase using monoclonal or polyclonal antibodies. 'Capture panning' of virus proteins from a homogenate of herpes simplex virus (IISV)-infected cells and selection of Phabs from an immunized phage library has resulted in the successful isolation of multiple Fab fragments specific for HSV glycoproteins ${ }^{4}$. Importantly, in a dimerized format, these antibodies exhibited neutralizing activity of live virus. This selection strategy removes the need for protein purification and coating under denaturing conditions and, because the mAb used for panning blocks the selection of phages against that particular epitope, allows the targeting of phages to pre-selected regions of the protein.

Several groups have reported on the selection of Phabs using intact eukaryotic cells in suspension or cells growing in mono- layers as targets ${ }^{5-7}$. Selection procedures on eukaryotic target cells can be extended by an absorption step in which the library is incubated with absorber cells to remove undesired specificities. For example, selection of phages for binding to malignant melanoma cells followed by absorption of eluted phages on normal melananocytes yielded antibodies that recognized the melanoma cells exclusively ${ }^{5}$.

Along this line, the combination of flow cytometry and phage-display-library technology has proved to be successful in isolating antibodies directed against surface markers present on subpopulations of cells in a heterogeneous mixture ${ }^{b}$. In this procedure, the phage library is incubated with the heterogeneous population of celis; the subpopulation of interest is subsequently stained with (combinations of) fluorochrome-labeled mAbs and sorted from the heterogeneous mixture by FACS (fluorescence-activated cell sorter) analysis. The nonselected cells in the mixture act as an absorber population, and the cell-sorter-based selection procedure in fact represents a very stringent washing step, resulting in the isolation of presumably higher-affinity antibodies.

We have applied this approach to evis blish large collections of $\mathrm{scFv}$ antibodies directed against cells of various hematopoietic lineages and differentiation stages, and to select phage antibodies directed against normal and malignant cells of endothelial and epithelial origin (T. Logtenberg, unpublished). Notably, these antibodies were isolated from a single, large synthetic phage-display library of $\mathrm{scF}_{\mathrm{V}}$ antibodies?. The power of this approach is illustrated by the selection of phages binding to the rare population of hematopoietic stem cells present in fetal bone marrow. Three rounds of flow-cytometric separation of as few as $10^{3}$ $\mathrm{CD} 34^{+} \mathrm{CD}^{-} 8^{-}$stem cells resulted in the isolation of a number of stem-cell-specific phages, including a phage that recognizes c-Kit t the receptor for stem cell factor, which is expressed on $\mathrm{CD} 34^{\text {bright }}$ hematopoietic precursor cells, CD34 dim pro-erythrocytes and perhaps mast-cell precursors (Fig. 1a) (J. de Kruif, unpublished). On the basis of tissue- and cellular-distribution patterns of target antigens recognized by phages selected by this approach, a sizeable proportion of 
these monoclonal Plabs appears to recognize novel, previously undetected cellsurface antigens. Finally, identification of novel target antigens by the screening of COS-cell-expression libraries with scFv antibodies is technically feasible".

Preparaticn of cells for phage selections may require treatment of tissues with proteolytic enzymes and/or cell culture, potentially resulting in the loss of phenotypic characteristics of the target cells. This notion is exemplified by murine thymic stromal cells: upon isolation and culture, these cells lose their characteristic phenotype asso ciated with the unique three-dimensional architecture of thymic stroma and, in addition, lose their ability to positively select differentiating $T$ cells (E.J. Jenkinson et al., pers. commun.). To circumvent th s problem, we have used intact fragments of freshly isolated whole thymic tissue as targets for phage selection using the synthetic library after pre-absorption with splenocytes and thymocytes After four rounds of selection on thymic fragments from different mouse strains, monoclonal Phabs were analyzed by immunohistochemical staining of frozen sections from a broad variety of murine tissues. From these phage selections, several scFv antibodies were obtained that bind to either the cell surface or intracellular proteins of thymic stroma. By performing consecutive phage selections on thymuses from different mouse strains, we appeared to obtain scFvs specific for evolutionary conserved epitopes, since some crossreacted with stromal cells in human thymus (Fig. 1b) (W. van Ewijk, unpublished). On the hasis of tissue-distribution and immunohistochemical-staining patterns, some of these monoclonal Phabs appear to recognize previously unknown structures on thymic stromal cells.

\section{Constructing antibody derivatives}

The antibody fragments obtained from phage-display libraries are monovalent, but multivalency is a desirable property in many in vitro and in vivo applications. In addition, linking two or more binding sites efficiently increases the functional avidity of antibody molecules. Bivalent (and bispecific) (scFv) ${ }_{2}$ and $\mathrm{F}(\mathrm{ab})_{2}$ fragments have been pro- duced successfully by association of two molecules through flexible linker polypeptides, chemical crosslinking and dimerization domains (reviewed in Ref. 10). scFv antibody fragments can be converted to dimers by genetic fusion of the scFv moiety to peptides derived from leucine-zipper protein interaction domains". Thus, modified scFv fragments dimerize spontaneously to bivalent ( $\mathrm{scFv})_{2}$ fragments in the bacterial periplasm. The use of both Fos and Jun permits the efficient production of bispecific (scFv) heterodimers". We have converted scFv antibodies from our synthetic library to intact Ig molecules by inserting them in eukaryotic expression constructs containing the C-region genes encoding each human lg isotype. These 'natural' antibodies that could be produced efficiently in cukaryotic cells, retained the specificity of the original $\mathrm{scF}$ and performed effector functions associated with the Fo portion (Fig. 1c) (E. Boel unpublished).

scFv fragments can be incorporated into liposomes to create immunoliposomes with specific cellular- or tissue-targeting properties. Addition of the E. coli lipoprotein signal sequence to $s \mathrm{Fr}$ fragments results in the stoichiometric in aivo biosynthetic lipid modification of $\mathrm{scFv}$. These strongly lipo philic lipo-scFv molecules efficiently anchor scFv fragments to membrane-encapsulated vesicles ${ }^{12}$. A CD22-specific scFv fragment isolated from our synthetic phage library was lipid modified and incorporated into liposomes. Immunofluoresient analysis of binding of CD22-immunoliposomes to blood leukncytes confirmed the specific binding of these vesicles to B-lineage cells (Fig. 1d).

\section{Concluding remarks}

Phage-display technology permits the selection of human antibody fragments from large libraries constructed from the $B$ cells of individuals or assembled in aitro from the genetic elements encoding antibodies. The procedure is extremely rapid and independent of the immunogenicity of the target antigen. An array of (subtractive) selection procedures on whole eukaryotic cells and intact tissues facilitates the isolation of antibodies against novel membrane molecules and epitopes, even when the target cells are ex- tremely rare or lose phenotypi, characteristics upon preparation. It can be envisaged that selection of Plabs in rico, in anlalugy with recently described experiments with phage-displayed peptides, will lead to the identification of novel organ-specific targeting molecules for therapy ${ }^{17}$. Engineering steps further allow the tailoring of antib.sdy fragments to meet specific needs in terms of avidity, pharmacokinetic properties and biological effector functions. These sharacteristics render this technology a valiable research tool and an approach to the production of human mAbs for immunotherapy. The large-scale production and :linical testing of these products poses a major challenge for the near future.

I. de Krnif, A-R. van der Vuurst de Vries, L. Cilenti, E. Boel ant T. Logtenberg (t.lugenbergolabazu.nl) are at the Dept of Immnuology, Lniadrity Hospifol Litrecht LItredit, The Netherhats; W. van Ezijk is at

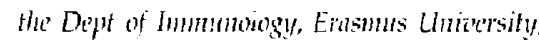
Rottrefam, the Nethothand:

\section{References}

1 Winter. G., Griffitlh, A.D., Hankins, R.E. and

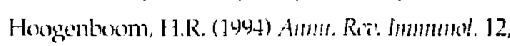
$4.33-15$

2 Burtom, D.R. and Barbas, C.F., III (1 (1994) Ads linumut. $37,191-280$

3 Vaughan, T.J., Williams, A.J., Pritchard, K. tt al. (1996) Nat. Bistecthol. 14, 309-314

4 Sanna, R.,. Williamson, R.A., De Logu, A. Bloom, F.E. and Burton, D.R. (1995) Proc. Nitl. Actut. Sri. U. S. A. 92, 6439-6443

5 Cai, X. and Garen, A. (1995) Pwc. Natt. Acat, Sci. U. S. A. $92,6537-6541$

6 de Kruif, J., Terstappen, L., Roel, E. and Logtenberg T. (1995) Proi: Natl. Actid. Sci. U. S. A. 92, 3938-3942

7 Zhang, H., Lake, D.F., Barbuto, J.A.M., Bernstein R.M., Grimes, W.J. and Hersh, E.M. (1995) Cimuer Res. 55, 3584, 3591

8 de Kruit, J., Boel, E. and Lontenburg, T. (1995)

1. Mol. Biol. 248, 97-115

9 de Kruif, J. and Logternber, T. (1996) I. Bind.

Cla'm. 271, 76301-7034

10 Holliger, P. and Winter, G. (1993) Cirrr. Opint.

Biotechnol. 4, 44-449

11 Pluckthum, A. (1992) Immmmol. Ror: 130, 151-188

12 Keinanen, K. and Laukkanen, M-L. (1994) FFBS

Liff. 346, 123-120

13 Pasqualini, R. and Ruoslaltit, E. (1996) Naturt

$380,364-366$ 